\title{
Kommentar zu Räume schaffen, Bedingungen und Kontexte organisieren
}

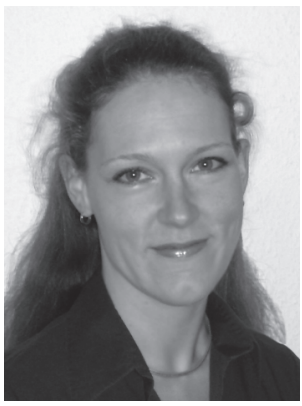

\section{Anna Katharina Liebscher}

Darüber, dass heutzutage Nachhaltigkeit fest in das Management zu integrieren ist, herrscht eigentlich Einigkeit. Die Frage nach dem wie ist diejenige, die die Forschung immer wieder vor Probleme stellt.

Die Annahme, dass die Verfolgung einer unternehmerischen Nachhaltigkeitsstrategie Menschen mit Nachhaltigkeits-Kompetenzen benötigt, setzt sich immer mehr durch - wie auch dieses Themenheft belegt. Nichtsdestoweniger scheint die Forschung in diesem Bereich zuweilen noch auf der Ebene des Darum-Wissens zu verweilen und die Ebene des Umsetzens im betrieblichen Umfeld zu umschiffen. Denn während die Frage, welche Kompetenzen eine nachhaltigkeitsorientierte Unternehmenskultur fördern, in der Forschung eine hochinteressante Fragestellung darstellt, interessiert die Führungskraft, die diese haben oder erlernen soll, doch eher die Konkretisierung der wissenschaftlichen Erkenntnisse.

Eine Weiterführung des Gedankens der Einflussnahme durch kulturelle Kompetenzen im Hinblick darauf, welche Kompetenzen denn nun diejenigen Fähigkeiten und Fertigkeiten beschreiben, die einer nachhaltigen Unternehmensausrichtung dienlich sind, wäre hier erstrebenswert. Oder geht es doch eher darum, überhaupt kulturelle Kompetenz zu besitzen, in der Annahme, dass diese der Nachhaltigkeit dienlich sein wird? Spielt die Zusammensetzung aus verschiedenen Bestandteilen dann überhaupt eine Rolle?

Unter der Voraussetzung, dass - wie die Autoren zu Beginn ganz treffend formulieren Reboundeffekte reine Effizienzmassnahmen auf den zweiten Blick als nicht-nachhaltig entlarven, bedeutet eine nachhaltigere Unternehmensausrichtung unglücklicherweise immer, mehr Geld, Zeit und Aufmerksamkeit investieren zu müssen. Hier scheint die wesentlich notwendige Kompetenz von Führungskräften und Entscheidern schon mittransportiert zu werden: die Fähigkeit, solche Anliegen, die dem primären Zweck der Erwirtschaftung von Gewinnen mit Hilfe möglichst effizienter Prozesse entgegenstehen, kommunizieren und legitimieren zu können. Auf diese Weise wird der Fokus bei der Beurteilung des eigenen Handelns von den Hauptwirkungen kultureller Prozesse auf deren Nebenwirkungen verschoben. Die Notwendigkeit eines solchen Vorgehens wird sehr deutlich, wenn sie anhand von Beispielen illustriert wird, wie die Autoren es anhand der fünf Herausforderungen gezeigt haben.

Die geforderte Berücksichtigung der Zeitlichkeit und der Wirkungen, die das ökonomische Handeln verursacht, wird noch schwieriger, sobald der Einzelakteur Unternehmen nicht nur sein eigenes Handeln betrachtet, sondern auch die Auswirkungen, die dieses auf seine Umwelten hat. Die Nachhaltigkeitskompetenz eines Einzelnen wird hier schnell die Grenzen ihrer Wirksamkeit erreichen. Gerade wenn eine Abkehr von der rein wettbewerblichen Tradition der Unternehmensführung gefordert wird, müssen solche Aspekte, die kooperatives Vorgehen begünstigen, als Bestandteil der Führungskompetenz betrachtet wer- 
den. Das Unternehmen als gesellschaftlicher Akteur ist eben doch stets eingebettet in sein gesellschaftliches Umfeld, zugleich aktiv im Wettbewerb und dem „Wohlwollen“ seiner Stakeholder ausgesetzt. Hiermit darf die Konzeption von Nachhaltigkeitskompetenz(en) nicht auf der individuellen Ebene halt machen, sondern muss auch die Fähigkeit, kollektive Prozesse mit verschiedenen Zielsetzungen, individuellen Präferenzen und unterschiedlichen Herangehensweisen an Probleme bisweilen sehr heterogener Akteure aushalten und gestalten zu können, beinhalten. Die Fähigkeit zur Koordination von Nachhaltigkeitsprozessen auf intra- und interorganisationaler Ebene wird damit zu einem zentralen Baustein der Nachhaltigkeitskompetenz.

Die Sicherung von Verwirklichungschancen sowohl der derzeitigen als auch künftiger Generationen entspricht hier dem Nachhaltigkeitsleitbild, das durch die Brundtland-Kommission der Nachhaltigkeitsdiskussion so viel Aufmerksamkeit beschert hat. Dieser Gedanke ist aus normativer Sicht unumstösslich. Gleichwohl werden für Unternehmen neben der ethischen Verpflichtung, einen Beitrag zu einer nachhaltigen gesellschaftlichen Entwicklung zu leisten, wohl immer auch rationale Gründe der Erhaltung des eigenen Unternehmens eine Rolle spielen müssen. Nur wenn der eigene Bestand sichergestellt wird, ist ein gesamtgesellschaftliches Engagement überhaupt möglich. Nachhaltigkeitskompetenz kann folglich nicht auf eine normative Komponente reduziert werden, sondern erfordert auch die Berücksichtigung von Ressourcenverfügbarkeiten - und zwar nicht von solchen Ressourcen, die derzeit existieren und beispielsweise durch die Ausübung von Macht von Wettbewerbern zu erhalten sind, wie die klassischen Ansätze postulieren, sondern von den in Zukunft benötigten Ressourcen. Die Orientierung am Gemeinschaftlichkeitsprinzip, die aufgrund der Einflussnahme zahlreicher Akteure auf diese grundsätzliche Verfügbarkeit von Ressourcen unausweichlich wird, und die Betrachtung des Umgangs mit commons scheinen ein vielversprechender Anfang für eine solche Entwicklung von kulturell geprägten, individuellen und kooperativen Nachhaltigkeitskompetenzen zu sein.

Anna Katharina Liebscher, Dr. rer. pol., ist wissenschaftliche Mitarbeiterin im Fachgebiet Nachhaltiges Management an der Universität Bremen.

Anschrift: Universität Bremen, Fachbereich Wirtschaftswissenschaft, Fachgebiet Nachhaltiges Management, Wilhelm-Herbst-Str. 12, 28359 Bremen, Tel.: +49 421/218-66784 Fax: +49 421/218-7422, E-Mail: liebscher@uni-bremen.de 